(C) 2007 IEEE. Reprinted, with permission, from Gabriel Aguirre-Ollinger, A 1-DOF assistive exoskeleton with virtual negative damping: effects on the kinematic response of the lower limbs

, Intelligent Robots and Systems, 2007. IROS 2007. IEEE/RSJ International Conference on, Oct-Nov 2007. This material is posted here with permission of the IEEE. Such permission of the IEEE does not in any way imply IEEE endorsement of any of the University of Technology, Sydney's products or services. Internal or personal use of this material is permitted. However, permission to reprint/republish this material for advertising or promotional purposes or for creating new collective works for resale or redistribution must be obtained from the IEEE by writing to pubspermissions@ieee.org. By choosing to view this document, you agree to all provisions of the copyright laws protecting it 


\title{
A 1-DOF Assistive Exoskeleton with Virtual Negative Damping: Effects on the Kinematic Response of the Lower Limbs
}

\author{
Gabriel Aguirre-Ollinger, J. Edward Colgate, Michael A. Peshkin and Ambarish Goswami
}

\begin{abstract}
We propose a novel control method for lowerlimb assist that produces a virtual modification of the mechanical impedance of the human limbs. This effect is accomplished through the use of an exoskeleton that displays active impedance. The proposed method is aimed at improving the dynamic response of the human limbs, while preserving the user's control authority. Our goal is to use active-impedance exoskeleton control to improve the user's agility of motion, for example by reducing the average time needed to complete a movement.

Our control method has been implemented in a 1-DOF exoskeleton designed to assist human subjects performing knee flexions and extensions. In this paper we discuss an initial study on the effect of negative exoskeleton damping (a particular case of active-impedance control) on the subject's time to complete a target-reaching motion. Experimental results show this effect to be statistically significant. On average, subjects were able to reduce the time to complete the motion by $16 \%$.
\end{abstract}

Index Terms-Rehabilitation robotics, exoskeleton, lower limb, active impedance, assist.

\section{INTRODUCTION}

We present a novel form of lower-limb assist, consisting of making the human limb interact with an exoskeleton that displays active mechanical impedance. Our overarching goal is to develop a control method for exoskeletons that provides sufficient flexibility to assist a wide variety of lower-limb motions, such as can be encountered in activities of daily living. Our approach to human assist is based on enhancing the kinematic response of the human limbs.

Exoskeleton designs can be classified in terms of their assistive capabilities as either passive or active devices. Exoskeletons that display passive behavior assist human users mainly by helping them employ their own muscle power more effectively, but do not actually supply energy to the user. In passive gravity support, an unactuated orthotic device can provide partial support of the user's weight by forming a mechanical path to the ground. Gravity balance of the freely-moving leg using springs has been implemented by Banala et al. [2]. Load-carrying assist is a special case of gravity support, typified by the BLEEX system [6], in which the exoskeleton supports a load carried by the user. The exoskeleton's controller uses positive kinematic feedback to scale up the device's mechanical admittance; however,

This project has been supported through a grant from the Honda Research Institute (Mountain View, CA).

Gabriel Aguirre-Ollinger (gaguirre@northwestern.edu), J. Edward Colgate and Michael A. Peshkin are with the Department of Mechanical Engineering, Northwestern University, Evanston, IL 60208, USA.

Ambarish Goswami is with the Honda Research Institute, Mountain View, CA 94041, USA. the device remains passive in its interaction with the user As complement to load-carrying capabilities, the system developed by Walsh et al. [13] uses passive devices like springs to store energy released during negative-work phases of the gait cycle, and releases it during the positive-work phases.

Our work focuses on active exoskeleton assist. An active device is one that can behave as a continuous energy source. Probably the most common approach to active assist is using the muscles' electromyographical (EMG) activity to control the actuators of the exoskeleton or orthosis. Rosen et al. [9] have developed a powered exoskeleton to assist elbow motion, using EMG as the primary command signal. Ferris [3] and his group have designed a pneumatically powered, myoelectrically controlled ankle-foot orthosis as a tool for human locomotor adaptation. The wearable robot HAL (Hybrid Assistive Limb) developed by Sankai et al. [5] includes four actuators for hip and knee assist, and uses feedback from both EMG and force sensors. A different form of active assist is represented by robotic gait trainers. The treadmill orthosis Lokomat [4] is typically used in position control mode to enforce a physiological gait trajectory on the patient. Lokomat can also be used in impedance control mode to adapt an abnormal gait pattern towards the desired one. The LOPES gait trainer [12] employs series-elastic actuation and force feedback to track a reference force; it has been used to control step height through virtual stiffness display [11].

\section{Human ASSist By ACTIVE IMPEDANCE}

Figure 1 shows a model of a 1-DOF exoskeleton designed to assist the motion of the knee joint. For the case of linear behavior, this device can be modeled as a LTI second-order rotational system.

Impedance control is used to regulate the interaction between the exoskeleton and the user. The exoskeleton's virtual impedance parameters consist of inertia moment $I_{e}^{d}$, damping $b_{e}^{d}$, and stiffness $k_{e}^{d}$. The superscript $d$ denotes that these are "desired" impedance values. Thus we define the exoskeleton's virtual impedance as

$$
Z_{e}^{d}(s)=I_{e}^{d} s+b_{e}^{d}+\frac{k_{e}^{d}}{s}
$$

For the exoskeleton to be truly assistive, it must be capable of making the transition from passive to active behavior. A physical system is said to be passive if the amount of energy $\Delta E_{\text {out }}$ that can be extracted from it over a certain period of time $\Delta t$ is never greater than the sum of the system's initial 


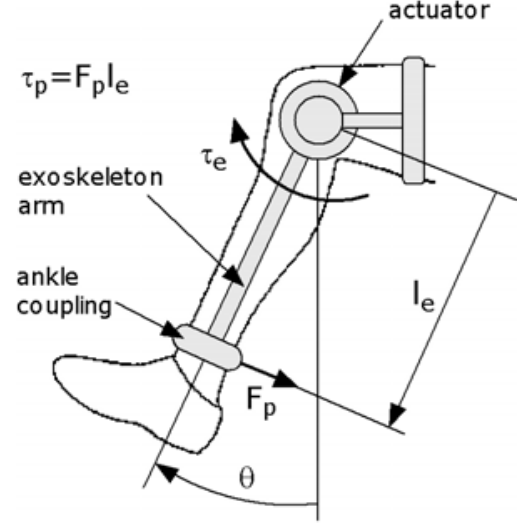

Fig. 1. Model of a 1-DOF exoskeleton for knee-joint assist.

energy $E_{o}$, plus the amount of energy $\Delta E_{i n}$ that entered the system during $\Delta t$ :

$$
\Delta E_{\text {out }} \leq E_{o}+\Delta E_{\text {in }}
$$

An active system, by contrast, is not subject to the above inequality. The exoskeleton can be used to produce a virtual modification of the human leg's impedance. The impedance of the human leg's shank, $Z_{h}(s)$, is characterized by inertia moment $I_{h}$, damping $b_{h}$, and stiffness $k_{h}$. We use the last term, $k_{h}$, mainly as a linearization of the gravitational torque acting on the leg, such that $m_{h} g l_{h} \sin \theta \approx k_{h} \theta$.

$$
Z_{h}(s)=I_{h} s+b_{h}+\frac{k_{h}}{s}
$$

Figure 2 shows the coupled system formed by the exoskeleton's virtual impedance and the human limb. For simplicity we are assuming the coupling between the exoskeleton and the human to be rigid. The term $\tau_{p}$ represents the net torque exerted by the exoskeleton on the human limb at the interaction port $p ; \tau_{h}^{a}$ is the net muscle torque under exoskeleton assist. It is easy to see that the equivalent impedance $Z_{h}^{d}(s)$ opposing the muscles' action is given by

$$
Z_{h}^{d}(s)=Z_{h}(s)+Z_{e}^{d}(s)
$$

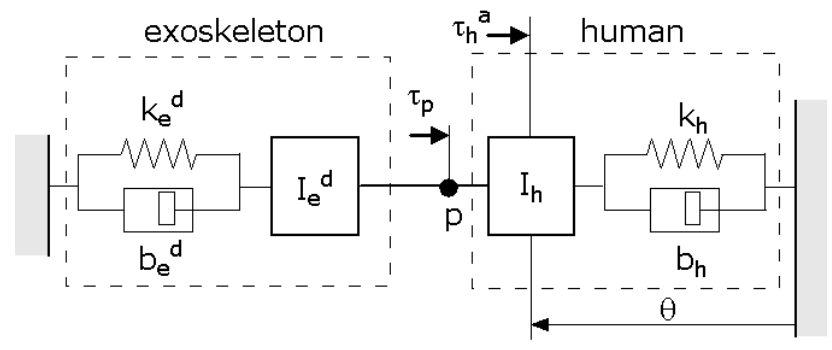

Fig. 2. Linear model of the coupled 1-DOF human-exoskeleton system.

Thus the dynamics of the coupled system are given by

$$
\left[\left(I_{h}+I_{e}^{d}\right) s+b_{h}+b_{e}^{d}+\left(k_{h}+k_{e}^{d}\right) \frac{1}{s}\right] \omega=\tau_{h}^{a}
$$

Equation (5) represents a generalized modification of the impedance parameters of the human limb segment by means of an exoskeleton. It suggests there is a wide range of possibilities to modify the relationship between the angular velocity trajectory $\omega$ and the net muscle torque $\tau_{h}^{a}$ by choosing the appropriate exoskeleton virtual impedance parameters $I_{e}^{d}, b_{e}^{d}$ and $k_{e}^{d}$.

We propose using the virtual modification of the lower limb's impedance as the primary source of human assist. The present study focuses on the use of negative exoskeleton damping, which is a particular case of active-impedance control. Because natural damping is an energy dissipation term, it makes sense to consider negative damping as a way to source energy from the exoskeleton to the user. Furthermore, since damping is a velocity-dependent effect, the exoskeleton-human interaction forces generated by negative damping become nearly zero when the leg is at rest or in quasi-static motion.

\section{IMPLEMENTATION OF A 1-DOF ADMITTANCE-CONTROLLED EXOSKELETON}

\section{A. Experimental platform: 1-DOF cable-driven exoskeleton}

For the actual implementation of the exoskeleton we have chosen and admittance control scheme: a torque sensor measures the interaction torque between the exoskeleton and the user, and uses it to issue a trajectory command. Our experimental platform is a 1-DOF exoskeleton mounted on a rigid base (Figure 3), designed to assist a person performing knee extensions and flexions. A custom-built ankle brace couples the user's leg to the exoskeleton arm. The arm's construction has been made as lightweight as possible in order to minimize its inertial effects. The ankle brace is mounted on a sliding bracket in order to accommodate any possible radial displacement of the ankle relative to the device's center of rotation.

The 1-DOF exoskeleton is designed for high backdriveability; to that purpose we have chosen to employ an AC servo motor with a large torque capability, and a low-ratio cable-drive transmission. The main advantage of the cable drive is the elimination of transmission backlash and friction, both of which can be a hindrance to impedance control, especially in the active region. The cable drive, shown in Figure 4, is similar in concept to that of the PHANToM haptic device [7]. A potential disadvantage of this type of transmission is axial cable compliance, which limits the bandwidth of the mechanism [10]. However, since typical lower-limb motions occur at low frequencies, the bandwidth requirements for this application are not particularly demanding. Figure 4 shows a detail of the exoskeleton's main assembly, consisting of the servo motor, the drive transmission and the exoskeleton arm. The motor is a brushless direct-drive AC servo with a power rating of $0.99 \mathrm{~kW}$ and a continuous torque rating of $2.0 \mathrm{Nm}$; it features an emulated encoder output of up to 32,768 counts before quadrature. The transmission ratio of the cable drive is $10: 1$, thus allowing a continuous torque output of $20.0 \mathrm{Nm}$. 


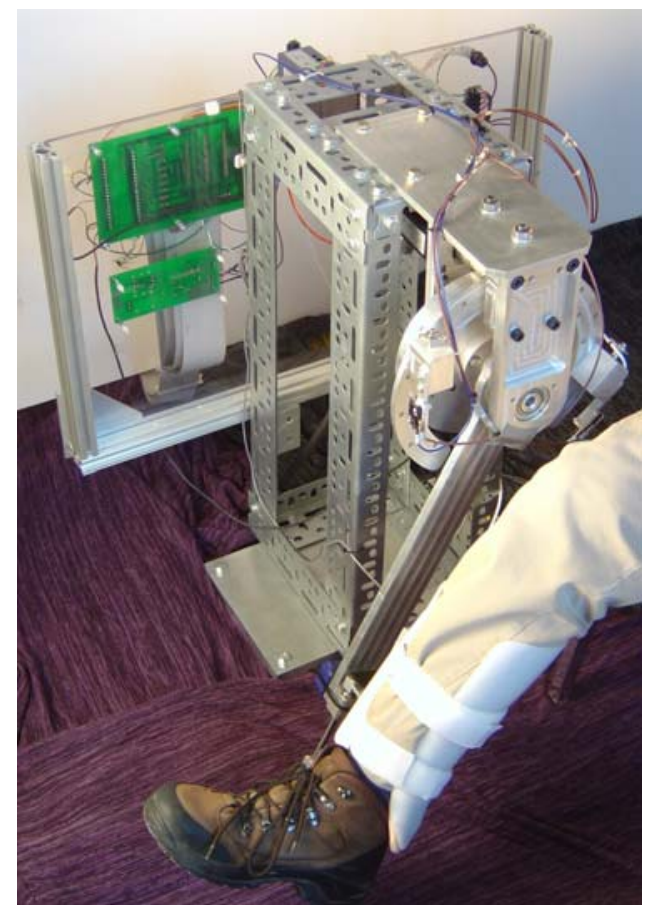

Fig. 3. 1-DOF exoskeleton for assisting knee-joint motion.

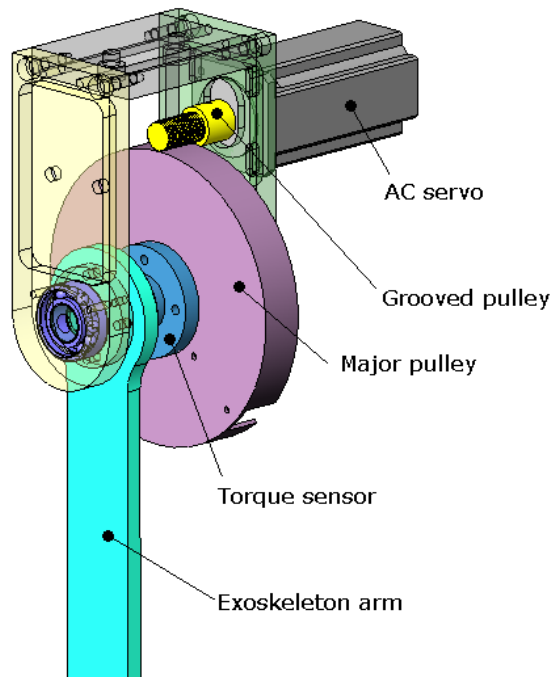

Fig. 4. Diagram of the 1-DOF exoskeleton's motor, drive and arm assembly.

\section{B. Exoskeleton controller implementation}

The dynamic response of the exoskeleton mechanism was estimated using an ARMAX model [8]. The system was excited by a white-noise torque signal applied to the servo, sampled at $1 \mathrm{kHz}$ and low-pass filtered at $30 \mathrm{~Hz}$. The output variable was the angular position, $\theta_{m}$. The experimental frequency response suggested that the exoskeleton mechanism can be modeled with sufficient accuracy using a secondorder linear model. Figure 5 shows a comparison between the measured frequency response and the response predicted by the model. For a measure of the quality of the model fit we used the variance-accounted-for (VAF) factor suggested
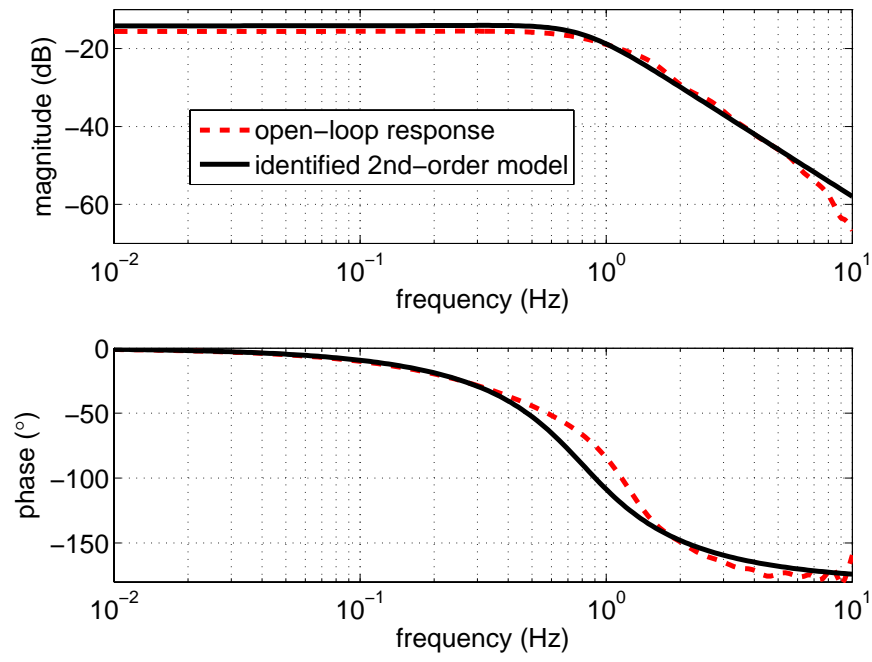

Fig. 5. Frequency response of the exoskeleton mechanism and second-order model fit.

by Veneman [12], which is defined as

$$
V A F=\left(1-\frac{\operatorname{var}(y-\hat{y})}{\operatorname{var}(y)}\right)
$$

where $y$ is the measured output and $\hat{y}$ is the output predicted by the model. The VAF for the mechanism was found to be $87.7 \%$, which indicates a high degree of system linearity. Thus the exoskeleton can be adequately controlled using a simple PID control scheme.

The general admittance control implementation for our exoskeleton is shown in Figure 6. The torque $-\tau_{p}$ exerted by the exoskeleton on the human limb is used as input to a virtual (desired) admittance block, that is, a control block containing the reciprocal of the exoskeleton's virtual impedance, $Z_{e}^{d}(s)$. The block generates a reference velocity trajectory, $\dot{\theta}_{\text {ref }}$. A complete kinematic reference trajectory is issued by integrating twice to obtain the reference angle $\theta_{\text {ref }}$ and its time integral; the latter is included in order to eliminate steady-state error. The reference trajectory is tracked by a closed-loop PID controller. A state observer is provided to compute an estimate of the actual velocity, $\dot{\theta}_{e s t}$, the actual angle, $\theta_{e s t}$, and its time integral. The main purpose of the observer is to eliminate the "encoder ripple" that typically occurs with low-velocity measurements. The control algorithm has been implemented in the QNX realtime operating system, with a sampling rate of $1 \mathrm{kHz}$.

The closed-loop frequency response of the controlled exoskeleton is shown in Figure 7. The input is the reference angle $\theta_{\text {ref }}$ and the output is the measured angle $\theta_{\text {est }}$. The current tuning of the PID controller provides maximum tracking accuracy over a bandwidth of about $4 \mathrm{~Hz}$. The trajectory-tracking fidelity of the controller can be estimated with the VAF metric by replacing $y$ with $\theta_{\text {est }}$ and $\hat{y}$ with $\theta_{\text {ref }}$. For a $2 \mathrm{~Hz}$ sinusoid, the tracking fidelity was found to be $99.29 \%$. Thus the admittance controller can accurately track angular trajectories in the typical frequency range of lower-limb motions. 


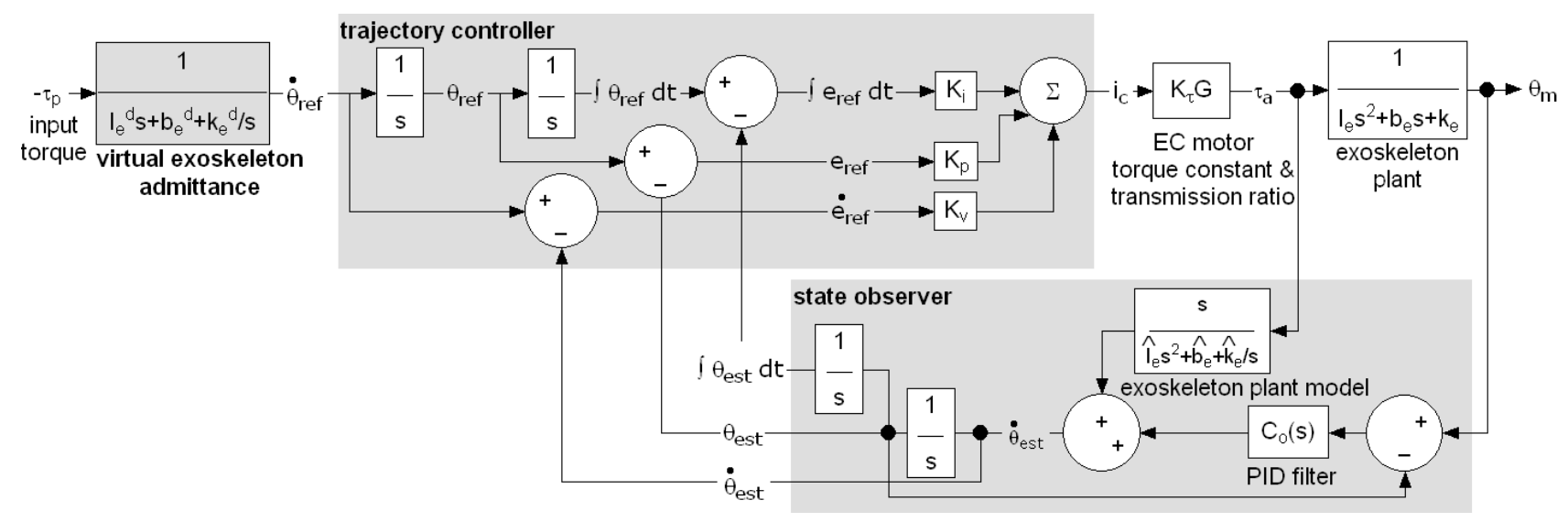

Fig. 6. Admittance control implementation for the 1-DOF exoskeleton.
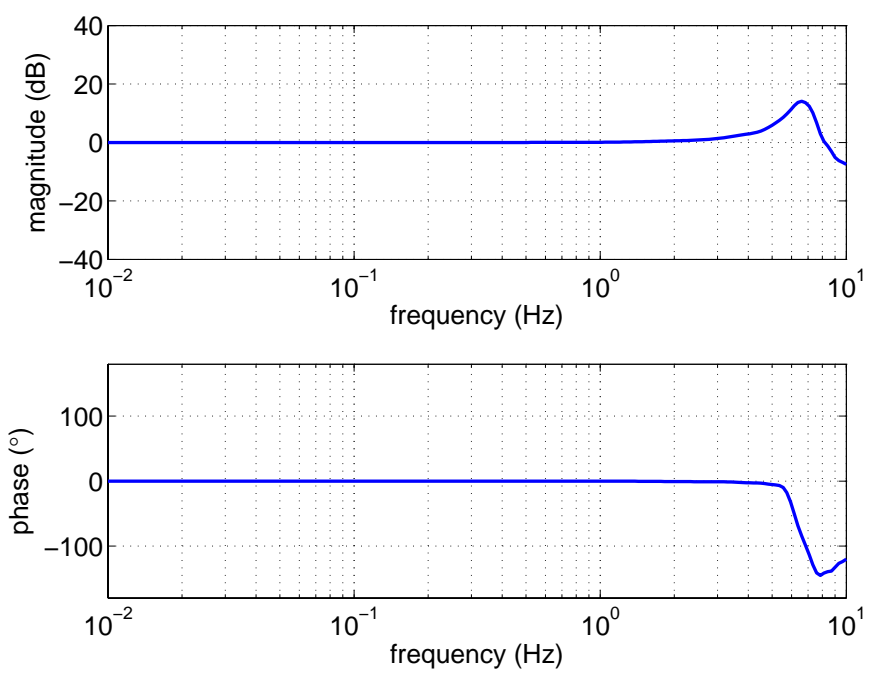

Fig. 7. Bode plot of the closed-loop frequency response from reference angle to measured angle.

\section{EXPERIMENTS WITH NEGATIVE EXOSKELETON DAMPING AND ITS EFFECT ON THE HUMAN LIMB}

\section{A. Negative damping regime: constant versus state- dependent}

We discuss now an experiment designed to test effect of negative exoskeleton damping on the step response of the lower leg. The hypothesis that negative damping can reduce the average time needed to complete a target-reaching movement. We have tested two regimes of negative damping, constant and state-dependent. Constant negative damping is the simplest regime to implement. However, while constant negative damping can help to initiate motion, it can also make it more difficult to stop since it cancels part of the natural human damping that can facilitate stopping the motion.

In the state-dependent damping regime, we use measurements of the kinematic state of the human limb to estimate the subject's motion intent, and modulate the exoskeleton's damping accordingly. A simple measure of intent is provided by the product of the measured angular velocity and the measured angular acceleration, $\dot{\theta}_{m} \ddot{\theta}_{m}$. This product equals the rate of change of kinetic energy of the human limb scaled by the limb's inertia. Assuming that the subject maintains control over the limb's motion at all time, a positive value of $\dot{\theta}_{m} \ddot{\theta}_{m}$ indicates intent to increase the speed of the limb. In this case the exoskeleton can assist the intended motion by making its virtual damping $b_{e}^{d}$ negative. Conversely, a negative $\dot{\theta}_{m} \ddot{\theta}_{m}$ indicates intent to decrease speed; thus the exoskeleton should reduce the magnitude of $b_{e}^{d}$ or even switch to a positive value thereof.

We have implemented the following function for statedependent exoskeleton damping:

$$
\begin{aligned}
& b_{e}^{d}\left(\dot{\theta}_{m}, \ddot{\theta}_{m}\right)= \\
& \quad B_{e}^{o}\left[\left(\frac{1+\delta_{e}}{2}\right) \tanh \left(\lambda_{e} \dot{\theta}_{m} \ddot{\theta}_{m}\right)+\left(\frac{1-\delta_{e}}{2}\right)\right]
\end{aligned}
$$

The rationale behind this function is that, for reasons of practicality and user safety, the magnitude of $b_{e}^{d}$ should be bounded. The damping coefficient $B_{e}^{o}$ is typically negative; the limit values of $b_{e}^{d}$ are thus $B_{e}^{o}$ and $-\delta_{e} B_{e}^{o}$, where $\delta_{e}$ is chosen from a range of values between -1 and 1 . The $\lambda_{e}$ coefficient is used to establish the rate at which the magnitude of the damping converges towards the extreme values. Currently the parameter values for the statedependent damping function are determined by trial and error, paying close attention to the users' reports on their ability to maintain control of their movements. In the present study it was observed that a transition from negative to positive damping felt unnatural to the users, due mainly to the large accelerations involved. Therefore it was decided to keep the exoskeleton's damping negative at all times, but decreasing in magnitude as $\dot{\theta}_{m} \ddot{\theta}_{m}$ becomes negative. Figure 8 shows a plot the actual state-dependent damping function used in the experiment. The maximum value of negative damping was $B_{e}^{o}=-1.2 \mathrm{Nms} / \mathrm{rad}$; the other parameter values were $\delta_{e}=-0.2$ and $\lambda_{e}=0.04$. 


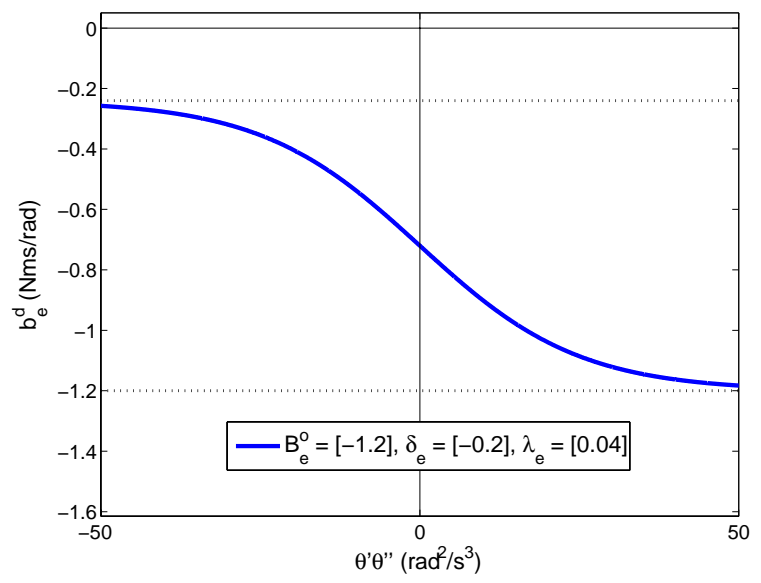

Fig. 8. Plot of a state-dependent damping function.

\section{B. Experimental protocol}

Five subjects (26-31 years of age; all healthy males) took part in the study. The Institutional Review Board of Northwestern University approved the experiments; all subjects gave informed consent before participation.

In our experiment, the subject moves the right shank while coupled to the exoskeleton. The exoskeleton's measured angular position is used to guide a virtual object on a computer screen towards a fixed target, as shown in Figure 9(a). The virtual object on the screen replicates the trajectory of the foot (Figure 9(b)), so that the center of its trajectory corresponds to the center of rotation of the knee. The virtual target is located on the path of the virtual object's trajectory. The target's width corresponds to a $8^{\circ}$ arc; its location is kept constant for this experiment $\left(60^{\circ}\right.$ from the vertical). The subject's task is to perform a target-reaching motion several times at different settings of negative damping.

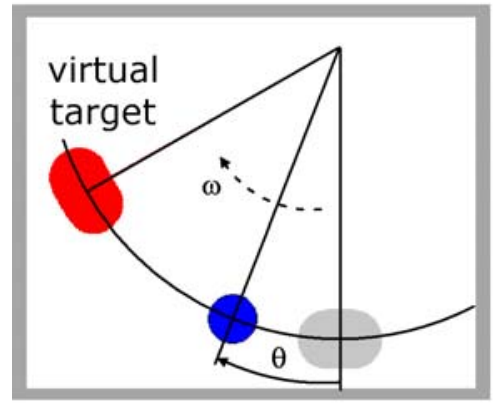

(a)

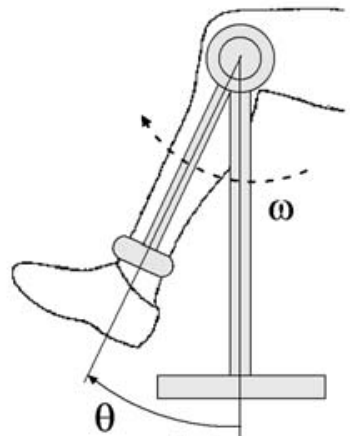

(b)
Fig. 9. Target-reaching task: (a) schematic diagram of the visual interface; (b) motion of the leg coupled to the exoskeleton.

Subjects were made to perform 3 series of target reaching motions under state-dependent exoskeleton damping, and 3 series under constant exoskeleton damping. For each series, 4 different levels of virtual damping $B_{e}^{o}$ were used: $0,-0.667$, -1.333 and $-2.0 \mathrm{Nms} / \mathrm{rad}$. In the case of state-dependent damping, $B_{e}^{o}$ corresponds to the maximum absolute value of the damping $b_{e}^{d}\left(\dot{\theta}_{m}, \ddot{\theta}_{m}\right)$ generated by the exoskeleton. In the constant-damping regime $b_{e}^{d}$ equals $B_{e}^{o} .10$ target-reaching motions were performed at each level of virtual damping. Thus the complete experiment consisted of a total of 240 motions.

Each motion begins with the shank in a vertical position $(\theta=0)$. A 2-second countdown is shown to the subject, after which the target appears on the screen. Subjects were instructed to try to establish a rhythm in their reaching motions with which they would be comfortable. The reaching motion is successful if the subject can keep the virtual object within the target for 0.05 seconds. A message on the screen informs the user whether the reaching motion was successful or failed. It must be emphasized that this experiment has no control over the visuomotor response on the subject, but only on the actual motion of the limb. In order to have a fixed reference for the onset of the limb's motion, time computation was initiated when the leg crossed a threshold angle of $2.5^{\circ}$ (end point of the "home" region) measured from the vertical.

\section{Statistical analysis of the experimental data}

The outputs of interest in this experiment are the time to reach the target and the overshoot of the target, defined as the difference between the most extreme position of the virtual object, and the angular position of the center of the target. The factors controlled for in this experiment are the level of negative damping $B_{e}^{o}$ and the number of the target-reaching motion series $i_{s}(1,2$ or 3$)$. The latter was included in order to determine whether learning took place during the experiment. The interaction between $b_{e}^{d}$ and $i_{s}$ was also considered. Only the data from successfully completed motions were included in the statistical analysis. We performed an analysis of variance (ANOVA) to test the significance of the experimental factors, and obtained the regression coefficients for those factors determined to be significant.

\section{EXPERIMENTAL RESULTS}

Figure 10 shows a comparison between target-reaching trajectories performed by one subject under both statedependent and constant exoskeleton damping; trajectories for the damping levels $B_{e}^{o}=-0.667$ and $B_{e}^{o}=-2.0 \mathrm{Nms} / \mathrm{rad}$ are shown for both damping regimes. The plots show the angular position, velocity and acceleration trajectories, as well as the time profile of the exoskeleton's damping. The shaded area (offset from the horizontal axis for clarity) represents the net amount of energy $\Delta E_{p}$ transferred from the exoskeleton to the user at the interaction port; the differences in the amount of energy transfer are readily apparent.

\section{A. Time to reach the target: effect of negative damping}

Table I shows the results of the statistical analysis of the experimental data, which includes the significance levels (p-values) of the factors, and the regression coefficients $\hat{\beta}_{i}$ for those factors deemed significant. The effect of negative damping on the time to reach the target was found to be 

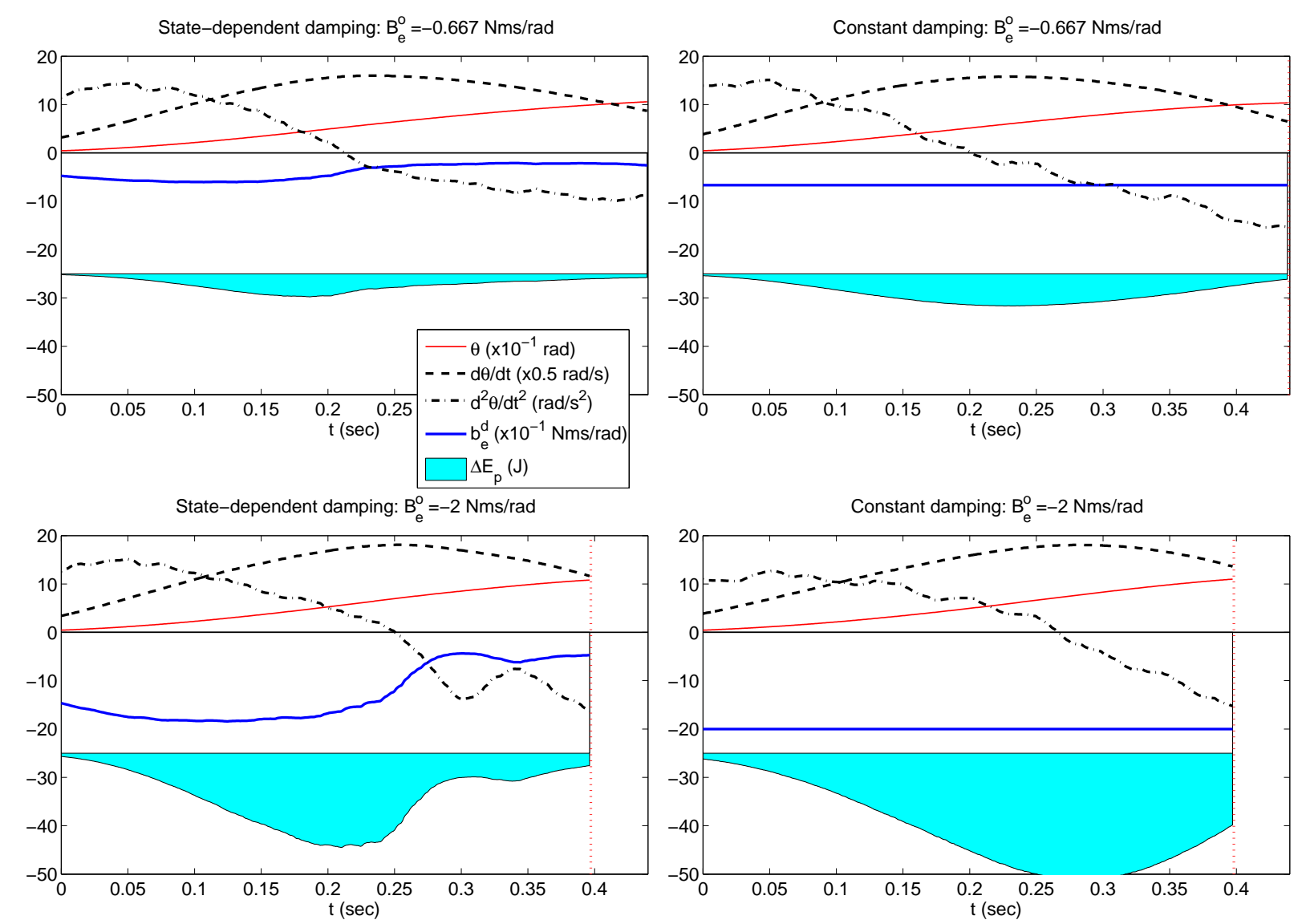

Fig. 10. Comparison of the time trajectories of target-reaching motions under state-dependent and constant damping.

highly significant $(P \simeq 0$ ). This effect is illustrated in Figure 11 which shows the results for one subject moving under the action of state-dependent damping. The plot shows the average time to reach the target, $\hat{t}_{T A R G}$, versus the exoskeleton's virtual damping level, $B_{e}^{o}$. Analysis of the complete data set from the experiment showed that the reduction in the average time $\hat{t}_{T A R G}$ was greater in the interval from 0 to $-1.333 \mathrm{Nms} / \mathrm{rad}$. This effect is presented in Table I: regression on only the first 3 damping levels ( 0 , -0.667 and $-1.333 \mathrm{Nms} / \mathrm{rad}$ ) yields a regression coefficient of 0.05673 , while the one obtained for all 4 damping levels is only 0.03643 . This suggests there may be an optimum damping level for the exoskeleton, beyond which the subjects essentially won't improve the time to reach the target.

Regression coefficients in Table I also show that statedependent damping in general has a larger effect on the time to reach the target than constant damping. A possible explanation is that, since constant negative damping makes it more difficult to stop the motion, subjects tend to increase their joint stiffness as a compensatory strategy, in detriment of the motion's speed. The average reduction in time to reach the target, contributed by state-dependent damping at its maximum value $\left(B_{e}^{o}=-2.0 \mathrm{Nms} / \mathrm{rad}\right)$ was estimated at $16 \%$. While this reduction is below the $29 \%$ level reported in the previous study [1], which involved one subject considerably familiarized with the device, it is remarkable in that

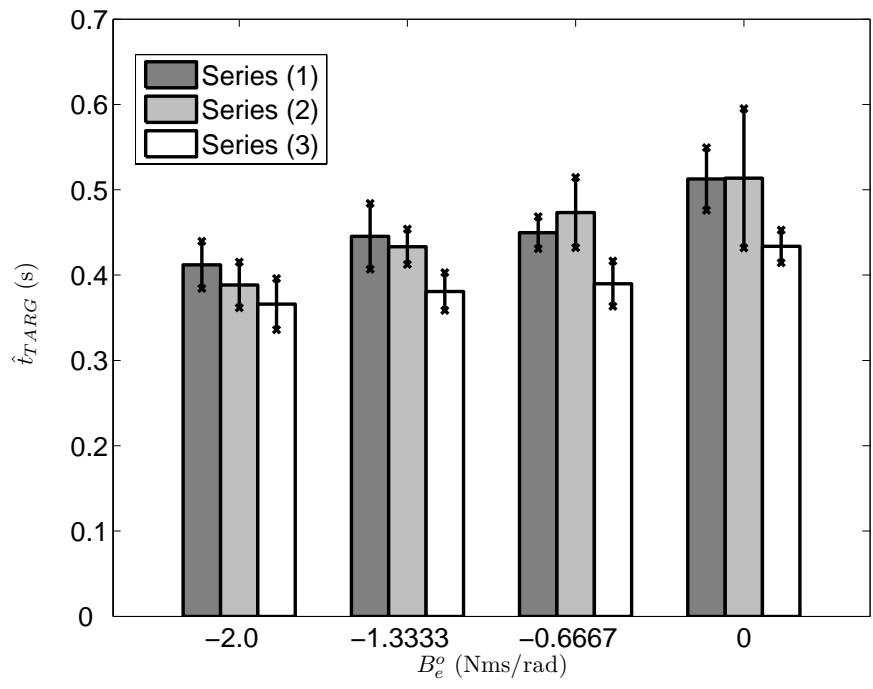

Fig. 11. Average time to reach target versus negative damping level: results for one subject moving under state-dependent damping.

subjects in this study had no previous experience using the exoskeleton. 
TABLE I

SIGNIFICANCE LEVELS AND REGRESSION COEFFICIENTS FOR THE TARGET-REACHING STUDY ( $B_{e}^{o}:$ DAMPING LEVELS, $i_{s}:$ SERIES NUMBER; $\hat{\beta}_{i}$ : REGRESSION COEFFICIENTS)

\begin{tabular}{|c|c|c|c|c|c|c|c|c|c|}
\hline \multirow{3}{*}{$\begin{array}{c}B_{e}^{o} \\
\text { levels }\end{array}$} & \multirow[t]{3}{*}{ Factor } & \multicolumn{4}{|c|}{ Time to reach target } & \multicolumn{4}{|c|}{ Overshoot } \\
\hline & & \multicolumn{2}{|c|}{$\begin{array}{c}\text { State-dependent damping } \\
b_{e}^{d}=b_{e}^{d}\left(\dot{\theta}_{m}, \ddot{\theta}_{m}, B_{e}^{o}, \delta_{e}, \lambda_{e}\right)\end{array}$} & \multicolumn{2}{|c|}{$\begin{array}{c}\text { Constant damping } \\
b_{e}^{d}=B_{e}^{o}\end{array}$} & \multicolumn{2}{|c|}{$\begin{array}{l}\text { State-dependent damping } \\
\text { (ditto) }\end{array}$} & \multicolumn{2}{|c|}{$\begin{array}{l}\text { Constant damping } \\
\text { (ditto) }\end{array}$} \\
\hline & & p-val. & $\overline{\beta_{i}}$ & p-val. & $\overline{\beta_{i}}$ & p-val. & $\overline{\beta_{i}}$ & p-val. & $\overline{\beta_{i}}$ \\
\hline All 4 & $\begin{array}{c}B_{e}^{O} \\
i_{s} \\
B_{e}^{o} \cdot i_{s}\end{array}$ & $\begin{array}{r}0 \\
0 \\
0.1298\end{array}$ & $\begin{array}{r}0.03643 \\
-0.02016\end{array}$ & $\begin{array}{r}0 \\
0 \\
0.0208\end{array}$ & $\begin{array}{r}0.02569 \\
-0.00893\end{array}$ & $\begin{array}{l}.1332 \\
0.0011 \\
0.2365\end{array}$ & $\begin{array}{r}-0.0035 \\
0.0051\end{array}$ & $\begin{array}{l}0.0004 \\
0.9787 \\
0.1271\end{array}$ & $\begin{array}{r}-0.0077 \\
0.0003\end{array}$ \\
\hline First 3 only & $\begin{array}{c}B_{e}^{O} \\
i_{s} \\
B_{e}^{o} \cdot i_{s}\end{array}$ & $\begin{array}{r}0 \\
0 \\
0.1854 \\
\end{array}$ & $\begin{array}{r}0.05763 \\
-0.02370\end{array}$ & $\begin{array}{r}0 \\
0 \\
0.0860 \\
\end{array}$ & $\begin{array}{r}0.04481 \\
-0.00585\end{array}$ & $\begin{array}{l}0.0012 \\
0.0511 \\
0.4211\end{array}$ & $\begin{array}{r}-0.0070 \\
0.0066\end{array}$ & $\begin{array}{l}0.7019 \\
0.0336 \\
0.0817\end{array}$ & $\begin{array}{r}-0.0057 \\
0.0010\end{array}$ \\
\hline
\end{tabular}

\section{B. Time to complete the target: effect of series number}

Table I also shows that the effect of the series number was statistically significant and negative, i.e. subjects tended to slightly increase their average target-reaching time from one test series to the next. The effect was more apparent for state-dependent damping than for constant damping, as suggested by the regression coefficients for $i_{s}$ alone. Thus the repetition of series of motions, as established in our protocol, can offset the improvement in reaching time contributed by negative damping. The effect of repetition of movements on the target-reaching time may be the result of two antagonistic factors, learning on one hand and fatigue on the other. A definite assessment of this effect will probably require longer series of movements at the same level of damping.

\section{Overshoot}

The average levels of overshoot (for successful motions) were basically uniform throughout the experiments. Computing the coefficients of determination for the controlled variables (subject, test number and damping) showed that these taken together contributed only $19 \%$ of the observed variations in overshoot. Thus the majority of the variations in overshoot can be attributed to random effects.

\section{CONCLUSIONS AND FUTURE WORK}

The experimental results support our hypothesis that negative damping contributes to reduce the time needed to complete a stereotyped lower-limb motion, although the effect of repetition needs to be better controlled. Reduction in average time was greater with state-dependent damping, which warrants further study of nonlinear damping functions. In this regard, a formal method for tuning the parameters of the state-dependent damping function needs to developed. Particularly, we want to be able implement our original concept of making the exoskeleton switch from being a power source (negative damping) to acting as a brake (positive damping), thus enabling it to help the user both to initiate and stop motion.

Compensatory motions, such as stepping to avoid a fall, usually involve above-average accelerations. Active exoskeleton impedance may be able to improve the subject's acceleration capability by increasing the motion bandwidth of the limb. We are planning to study the effects of active exoskeleton impedance on movements executed in response to unpredictable stimuli. The results may provide insight into the potential effect of a wearable exoskeleton on the user's response to external perturbations.

\section{REFERENCES}

[1] G. Aguirre-Ollinger, J. Colgate, M. Peshkin, and A. Goswami. Active impedance control of a lower-limb assistive exoskeleton. Proceedings of the IEEE International Conference on Rehabilitation Robotics, pages 188-195, 2007.

[2] S.K. Banala, S. K. Agrawal, A. Fattah, V. Krishnamoorthy, W.-L. Hsu, J. Scholz, and K. Rudolph. Gravity-balancing leg orthosis and its performance evaluation. IEEE Transactions on Robotics, 22(6):12281239, 2006.

[3] D.P. Ferris, J.M. Czerniecki, and B. Hannaford. An ankle-foot orthosis powered by artificial pneumatic muscles. Journal of Applied Biomechanics, 21:189-197, 2005.

[4] S. Jezernik, G. Colombo, and M. Morari. Automatic gait-pattern adaptation algorithms for rehabilitation with a 4-dof robotic orthosis. IEEE Transactions on Robotics and Automation, 20(3):574-582, 2004.

[5] H. Kawamoto, Suwoong Lee, S. Kanbe, and Y. Sankai. Power assist method for HAL-3 using EMG-based feedback controller. IEEE International Conference on Systems, Man and Cybernetics, 2:1648$1653,2003$.

[6] H. Kazerooni, J.-L. Racine, L. Huang, and R. Steger. On the control of the Berkeley Lower Extremity Exoskeleton (BLEEX). IEEE International Conference on Robotics and Automation, pages 43534360, April 2005.

[7] H.Y.K. Lau and L.C.C. Wai. Implementation of position force and position position teleoperator controllers with cable driven mechanisms. Robotics and Computer-Integrated Manufacturing, 21(2):145152, 2005.

[8] L. Ljung. System Identification - Theory For the User. PTR Prentice Hall, 2 edition, 1999.

[9] J. Rosen, M. Brand, M.B. Fuchs, and M. Arcan. A myosignal-based powered exoskeleton system. IEEE Transactions on Systems, Man and Cybernetics, 31(3):210-222, 2001.

[10] W.T. Townsend and J.A. Guertin. Teleoperator slave - WAM design methodology. Industrial Robot, 26(3):167-177, 1999.

[11] E.H.F. Van Asseldonk, R. Ekkelenkamp, J.F. Veneman, F.C.T. Van der Helm, and H. Van der Kooij. Selective control of a subtask of walking in a robotic gait trainer(LOPES). Proceedings of the IEEE International Conference on Rehabilitation Robotics, pages 841-848, 2007.

[12] J.F. Veneman, R. Ekkelenkamp, R. Kruidhof, F.C.T. Van der Helm, and $H$. Van der Kooij. A series-elastic and bowden-cable-based actuation system for use as torque-actuator in exoskeleton-type training. The International Journal of Robotics Research, 25(3):261-281, 2006.

[13] C.J. Walsh, D. Paluska, K. Pasch, W. Grand, A. Valiente, and H. Herr. Development of a lightweight, underactuated exoskeleton for loadcarrying augmentation. Proceedings of the IEEE International Conference on Robotics and Automation, pages 3485-3491, 2006. 